\title{
Fiber Optic Transceiver Qualification for Space Applications
}

Chuck Tabbert

ctabbert@ultracomm-inc.com

(505) 400-4785

www.ultracomm-inc.com 


\section{Market Use Issues}

- Significant interest in using fiber optic interconnects for high speed intra-satellite communication

- On Orbit now -

2.5G per channel(4x4) configuration - SNAP12 package

- In qualification 10G per channel $(4 \times 4)$ configuration - QFN package

- In initial production - $25 \mathrm{G}$ per channel (4x4) configuration - Chip-Scale-Package (CSP) with commercial driver ASIC

- Fiber optic transceivers should be treated as hybrid with light interface

- Focus on the reliability of the lasers (single or multi-mode) and cable interface to module (coupling losses in harsh environments)

- Standard qualification procedures are being truncated by customers for specific mission profiles

- Too Long

- Too Costly

- Not sure failure mechanisms have been properly excited

- New SAE publication - ARP 6318 - Initial testing assessment for fiber optic components

- Geared towards avionic applications (no radiation testing included)

- Take it to break it - testing to failure 


\section{Real-World Case Study}

- US National Asset satellite program has baselined 40G modules for use

- Standard Space Hybrid qual with particular focus on laser (VCSEL) reliability and manufacturing handling procedures

- Implemented two specific laser tests - Post laser attach

- Test 1 - Leakage Test Reverse Biased

- Standard VCSEL test with derived mission limits

- Test 2 - Optical Spectral Bandwidth

- Using CERN Measure-Stress-Measure Method

- Tight Delta parameters on VCSEL currents pre and post burn-in

- Full battery of radiation tests at module and semiconductor level

- Total Dose

- ELDRS on SiGe ASIC

- Single Event Upset (SEU)

- Proton

- Latch-Up

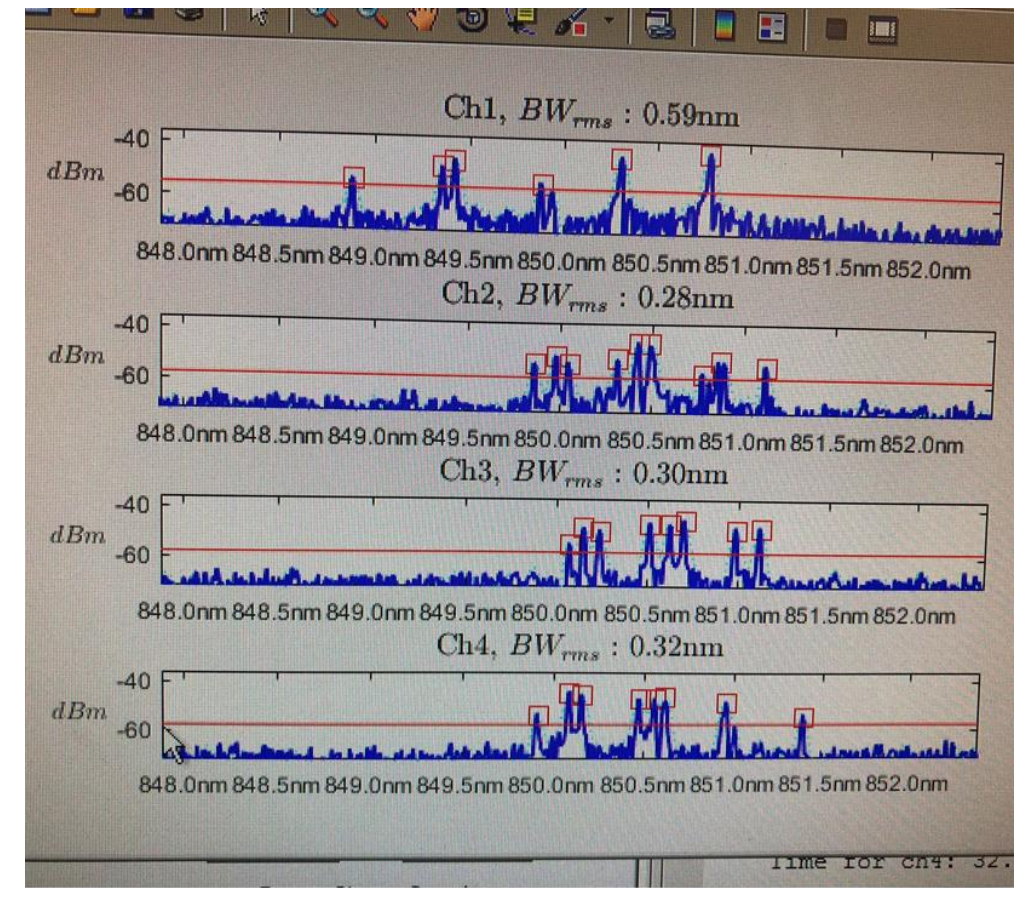

- Completed Worst Case Analysis (WCA) \& Failure Modes and Effects Analysis (FMEA)

- Customer performed extensive mission profile analysis to bound laser biasing conditions at desired data rate to insure desired life expectancy

- Reliability models (ie Hnbk 217, Accelerated Aging Models) too pessimistic 